\title{
Multimodal Non-rigid Warping for Correction of Distortions in Functional MRI
}

\author{
Pierre Hellier and Christian Barillot \\ IRISA, INRIA-CNRS unit, Campus de Beaulieu, F-35042 Rennes cedex, France. \\ \{phellier,barillot\}@irisa.fr
}

\begin{abstract}
This paper deals with the correction of distortions in EPI acquisitions. Echo-planar imaging (EPI) data is used in functional resonance imaging (fMRI) and in diffusion tensor MRI (dMRI) because of its impressive ability to collect data rapidly. However, these data contain geometrical distortions that degrade the quality of the scans and disturb their interpretation. In this paper, we present a fully automatic 3D registration algorithm to correct these distortions. The method is based on the minimization of a cost function (including mutual information and regularization) with a hierarchical multigrid optimization scheme. We present a numerical evaluation on simulated data and results on real data.
\end{abstract}

keywords: registration, multimodal fusion, EPI distortions, fMRI, unwarping.

\section{Introduction}

\subsection{Context}

To explore the brain, on its anatomical and functional sides, different modalities are now commonly used in clinical diagnosis and therapy planning. We distinguish two types of images: anatomical images (MRI, CT, angiography) and functional images (fMRI, PET, SPECT, MEG, EEG). These acquisitions measure different anatomical or physiological properties within the patient that are not redundant but complementary. Therefore, these images must be aligned (registered) so that no information is excluded from the diagnosis and therapeutic processes. To register these images, a rigid (translation, rotation and scaling) transformation is generally sufficient because the volumes are acquired from the same patient (see $[13,14]$ for tutorials). Nevertheless, we might have to perform non-rigid registration if there are distortions in one acquisition.

Among the functional images of the brain, fMRI is an appealing technique because it offers a good tradeoff between spatial and temporal resolution. To increase its temporal resolution, echo-planar imaging (EPI) is used because it makes possible to collect at least five slices per second at a reduced spatial resolution. The drawback of this impressive acquisition rate is that it may introduce artefacts and distortions in the data. More details about these distortions can be found in [11].

If the distortions do not vary during the time series, they will not affect much the detection of subtle signal changes, but they will perturb the localization of the functional activity once being overlapped to the anatomical volume. It becomes necessary to correct these geometrical distortions in order to accurately identify activated areas. 


\subsection{Related Work}

Many research has been made to develop algorithm that perform automatic rigid alignment of multimodal images. Earliest methods rely on the extraction and matching of singular structures (fiducials, curves or surfaces). The major problem is the extraction of the attributes to be matched, and the precision/robustness with respect to this extraction. "Voxel-based" methods are overwhelming "feature-based" methods as they are automatic and more accurate [22]. "Voxel-based" methods rely on the maximization of a similarity measure between two volumes.

Several similarity measures have been proposed: Woods et al. [23] proposed a similarity measure based on the local comparison of $2 n d$ order moments. Collignon et al. [3] and Viola et al. [21] proposed simultaneously to use mutual information, a statistical measure of the dependence between two distribution based on information theory. Studholme et al. [18] presented an overlap invariant version of the mutual information. More recently, Roche et al. [17] presented a method based on the maximization of the correlation ratio.

Although many efforts have been made to perform rigid registration, as far as we know, there has been few research concerning non-rigid multimodal registration. Two radically different approaches have been proposed:

The first approach consists in correcting the inhomogeneity field with the phase information $[8,1,10]$. The phase of the raw MR signal (k-space signal) is generally not used since we only need the amplitude to construct the images. The dominant distortion caused by eddy currents may be considered to be a scaling and, in the phase encoding direction, a shear and a translation. This information can then used to correct the recorded signal. These methods require to have the phase information, which is a constrain for almost all MR equipments. Furthermore, these methods are designed to correct only eddy currents-induced artefacts, which is not the only source of distortions.

Another way of considering the problem is the computer vision point of view, where the goal is to design a non-rigid multimodal registration method that can compensate for the EPI distortions. In this category, we distinguish two approaches: Maintz et al. [12] and Gaens et al. [6] proposed an algorithm that seek a non-rigid transformation by maximization of mutual information. They use a "block-matching" minimization scheme with a gaussian filtering of the estimated deformation field to avoid blocky effects. On local windows, the estimation does not take into account the spatial context of the deformation field and only a translation is estimated. Furthermore, these methods are only performed in $2 D$.

An interesting approach is described in [7]. This method considers the multimodal registration problem as a monomodal registration problem, and therefore estimates alternatively an intensity correction and a monomodal registration. This method dramatically depends on the intensity correction scheme and for these reasons, the iterative algorithm is not proved to be stable.

\subsection{General Description of the Method}

In this paper we propose a method that does not require pre-processing, nor phase acquisition, and we do not estimate any intensity correction. After rigid registration, we 
estimate a deformation field by minimizing a cost function that is composed of two terms: a similarity measure and a regularization term in order to ensure spatial coherence of the deformation field. We also use a minimization procedure described in [9]. We design a multigrid minimization scheme that is flexible, efficient and simple.

This paper is organized as follows: we describe successively the rigid registration step, the formulation of the problem and the multigrid minimization scheme. Then we present an evaluation of the method on simulated data and results on real data.

\section{Non-rigid Multimodal Registration Method}

\subsection{Rigid Registration Step}

To initialize the algorithm, we perform a rigid registration step. We estimate a rigid transformation that maximizes the mutual information. Given two discrete random variables $A$ and $B$ and their marginal probability distribution $p_{A}(a)$ and $p_{B}(b)$, let us note $p_{A, B}(a, b)$ the joint distribution. Mutual information $I(A, B)$ is then defined as [3,21]:

$$
I(A, B)=\sum_{a, b} p_{A, B}(a, b) \frac{p_{A, B}(a, b)}{p_{A}(a) p_{B}(b)}=H(A)+H(B)-H(A, B),
$$

with

$$
H(A)=-\sum_{a} p_{A}(a) \log _{2}\left(p_{A}(a)\right) \text { and } H(A, B)=-\sum_{a, b} p_{A, B}(a, b) \log _{2}\left(p_{A, B}(a, b)\right) .
$$

We choose an arbitrary world coordinate system, whose anatomical orientation is known, and in which the center of the axis correspond to the center of the volume, with a voxel size of $1 \mathrm{~mm}$. The transformation $T$ that maps the floating volume $B$ (EPI acquisition) onto the reference volume $A$ (anatomical volume) is estimated in the world coordinate system.

The registration is performed through a multiresolution optimization scheme (construction of a pyramid of volumes by successive isotropic filtering and subsampling in each direction). At each resolution level, the similarity $I(A, T(B))$ is maximized w.r.t. the parameters of the transformation using a Powell's algorithm [16]. We calculate the joint histogram on the overlapping part of $A$ with $T(B)$ by partial volume interpolation, the latter being known as providing a smooth cost function. Let us note $T_{0}$ the final rigid transformation.

\subsection{An Energy-Based Formulation}

To compensate for local geometrical distortions, a $3 D$ deformation field $\boldsymbol{w}$ must then be estimated. Let us note $T_{w}$ the transformation associated with the deformation field $\boldsymbol{w}$. The total transformation $T_{w} \circ T_{0}$ maps the floating volume onto the reference volume $A$. The field $\boldsymbol{w}$ is defined on $S_{B}$, where $S_{B}$ denotes the voxel lattice of volume $B$.

In a Bayesian context, we formulate the registration problem with gibbs prior as the minimization of a cost function: 


$$
U\left(\boldsymbol{w} ; A, B, \mathrm{~T}_{\mathbf{0}}\right)=-I\left(A,\left(\mathbf{T}_{\boldsymbol{w}} \circ \mathrm{T}_{\mathbf{0}}\right)(B)\right)+\alpha \sum_{<s, r>\in \mathcal{C}_{B}}\left\|\boldsymbol{w}_{s}-\boldsymbol{w}_{r}\right\|^{2},
$$

where $\mathcal{C}_{B}$ is the set of neighboring pairs of volume $B$ (if we note $\mathcal{V}$ a neighborhood system on $S_{B}$, we have: $\left\langle s, r>\in \mathcal{C}_{B} \Leftrightarrow s \in \mathcal{V}(r)\right.$ ), and $\alpha$ controls the balance between the two energy terms. The second term is a spatial regularization term that ensures the coherence of the deformation field.

\subsection{Multigrid Minimization}

Motivations The direct minimization of equation (1) is impossible for different reasons: if we estimate iteratively the deformation field on very small regions (the region could eventually be reduced to a voxel), mutual information will be inadequate, because the entropy measure is only meaningful for large groups of voxels. Furthermore, the algorithm will be extremely time-consuming, because the propagation of the regularization will be limited to small regions, and thus very slow. Finally, we need to specify a model to be estimated for the deformation field.

To overcome these difficulties (that are classical in computer vision when minimizing a cost function involving a large number of variables), multigrid approaches have been designed and used in the field of computer vision $[5,15,19]$. Multigrid minimization consists in performing the estimation through a set of nested subspaces. As the algorithm goes further, the dimension of these subspaces increases, and the estimation becomes more and more accurate. In practice, the multigrid minimization usually consists in choosing a set of basis functions and estimating the projection of the "real" solution on the span of the set of basis functions.

Description We use a multigrid minimization based on successive partitions of the initial volume, which is an extension of our previous work [9]. At each grid level $\ell$, corresponding to a partition of cubes, we estimate an incremental deformation field $\mathrm{d} \boldsymbol{w}^{\ell}$ that refines the previous estimation $\boldsymbol{w}^{\ell}$. Let us note $T_{\ell}$ the transformation associated with the incremental deformation field $\mathrm{d} \boldsymbol{w}^{\ell}$.

At grid level $\ell, \Xi_{\ell}=\left\{\Xi_{n}, n=1 \cdots N_{\ell}\right\}$ is the partition of the volume $B$ into $N_{\ell}$ cubes $\Xi_{n}$. A 12-dimension parametric deformation field is estimated on each cube $\Xi_{n}$, therefore the total increment deformation field $\mathrm{d} \boldsymbol{w}^{\ell}$ is piecewise affine. Contrary to block-matching algorithms, we have an interaction between the cubes of the partition, so that we do not have "block-effects" in the estimation.

At coarsest level $\ell_{c}$, the partition is a regular one, with cubes of size $2^{3 \ell_{c}}$. When we change of grid level, each cube is regularly divided and we stop at grid level $\ell_{f}$. Please note that there is no need to have a regular subdivision, it may be adaptive (see [9]) and constrained by functional ROI for instance. The final transformation $T_{\boldsymbol{\ell}_{f}} \circ \ldots \circ \mathrm{T}_{\boldsymbol{\ell}_{c}} \circ \mathrm{T}_{\mathbf{0}}$ expresses the hierarchical decomposition of the deformation field.

Estimation At grid level $\ell$ and on each cube $\Xi_{n}$, we estimate an affine displacement increment defined by the parametric vector $\Theta_{n}^{\ell}: \forall s=(x, y, z) \in \Xi_{n}, \mathrm{~d} \boldsymbol{w}_{s}=P_{s} \Theta_{n}^{\ell}$, with $P_{s}=\mathbb{I}_{3} \otimes\left[\begin{array}{llll}1 & x_{s} & y_{s} & z_{s}\end{array}\right]$ (operator $\otimes$ denotes the Kronecker product). Let us note 
$\mathrm{T}_{\Theta_{n}^{\ell}}$ the transformation associated with the parametric field $\Theta_{n}^{\ell}$. We have $\mathrm{T}_{\ell}=\mathrm{T}_{\mathrm{d} \boldsymbol{w}^{\ell}}$ and $\mathrm{T}_{\Theta_{n}^{\ell}}=\mathrm{T}_{\mathrm{d} \boldsymbol{w}^{\ell} \mid \Xi_{n}}$, where $\mathrm{T}_{\mathrm{d} \boldsymbol{w}^{\ell} \mid \Xi_{n}}$ denotes the restriction of $\mathrm{T}_{\Theta_{n}^{\ell}}$ to the cube $\Xi_{n}$.

A neighborhood system $V^{\ell}$ on the partition $\Xi_{\ell}$ derives naturally from $\mathcal{V}$ (section 2.2):

$\forall n, m \in\left\{1 \cdots N_{\ell}\right\}, m \in V^{\ell}(n) \Leftrightarrow \exists s \in \Xi_{n}, \exists r \in \Xi_{m} / r \in \mathcal{V}(s)$. $\mathcal{C}$ being the set of neighboring pairs on $S^{k}$, we must now distinguish between two types of such pairs: the pairs inside one cube and the pairs between two cubes:

$\forall n \in\left\{1 \ldots N_{\ell}\right\},<s, r>\in \mathcal{C}_{n}^{\ell} \Leftrightarrow s \in \Xi_{n}, r \in \Xi_{n}$ and $r \in \mathcal{V}(s)$.

$\forall n \in\left\{1 \ldots N_{\ell}\right\}, \forall m \in V^{\ell}(n),<s, r>\in \mathcal{C}_{n m}^{\ell} \Leftrightarrow m \in V^{l}(n), s \in \Xi_{n}, r \in \Xi_{m}$ and $r \in$ $\mathcal{V}(s)$.

With these notations, at grid level $\ell$, the cost function (1) can be modified as:

$$
\begin{aligned}
& \stackrel{\star}{U}\left(\Theta^{\ell} ; A, B, \mathbf{T}_{\mathbf{0}}, \boldsymbol{w}^{\ell}\right)=-\sum_{n=1}^{N_{\ell}} I\left(A,\left(\mathbf{T}_{\Theta_{n}^{\ell}} \circ \mathbf{T}_{\boldsymbol{w}^{\ell}} \circ \mathrm{T}_{\mathbf{0}}\right)\left(B_{\mid \Xi_{n}}\right)\right) \\
& +\frac{\alpha}{2} \sum_{n=1}^{N_{\ell}}\left[\sum_{m \in V^{\ell}(n)} \sum_{<s, r>\in \mathcal{C}_{n m}^{\ell}}\left\|\left(\boldsymbol{w}_{s}^{\ell}+P_{s} \Theta_{n}^{\ell}\right)-\left(\boldsymbol{w}_{r}^{\ell}+P_{r} \Theta_{m}^{\ell}\right)\right\|^{2}\right] \\
& +\alpha \sum_{n=1}^{N_{\ell}}\left[\sum_{<s, r>\in \mathcal{C}_{n}^{\ell}}\left\|\left(\boldsymbol{w}_{s}^{\ell}+P_{s} \Theta_{n}^{\ell}\right)-\left(\boldsymbol{w}_{r}^{\ell}+P_{r} \Theta_{n}^{\ell}\right)\right\|^{2}\right],
\end{aligned}
$$

where $B_{\mid \Xi_{n}}$ denotes the restriction of volume $B$ to the cube $\Xi_{n}$. The minimization is performed with an ICM algorithm (each cube is iteratively updated while its neighbors are "frozen"). On each cube, Powell's algorithm is used to estimate the parametric affine increment.

\section{Results}

\subsection{Simulated Data}

To evaluate the multimodal registration method, we use the simulated database of the MNI (Brainweb :http://www.bic.mni.mcgill.ca/brainweb) [4]. The T1weighted MR volume is the reference volume ( $3 \%$ noise and $9 \%$ inhomogeneity), whereas the T2-weighted MR volume is the floating volume.

From the T2-weighted MR volume, we extract a sub-volume and we apply a rigid transformation (3 rotations and 3 translations). To simulate local geometrical distortions, we apply a Thin Plate Spline [2] deformation to the volume. The thin plate deformation is computed by choosing one point in the volume and a displacement for this point. We choose a displacement of magnitude 5 voxels, with no privileged direction. Furthermore, the thin-plate deformation field is constrained to be naught at the border of the volume.

After rigid registration (see figure 1), distortions are clearly visible. On the axial view, ventricles are not well registered ; on the sagittal and coronal view, the sagittal mid-plane is not well aligned. We then perform the multigrid non-rigid registration 
from grid level 7 until grid level 5 to avoid useless computational efforts. After nonrigid registration, the internal structures are accurately registered (see ventricles on the axial view for instance).
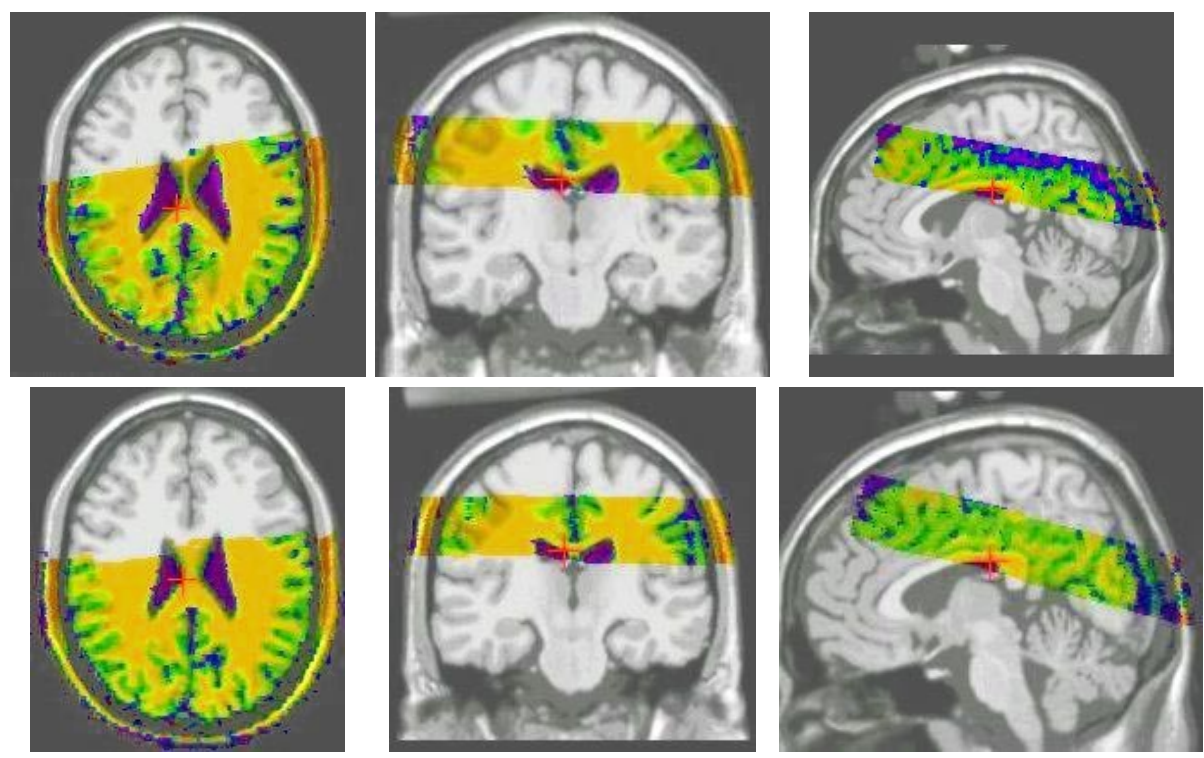

Fig. 1. Results of the registration on simulated data. Top: results after rigid registration. Distortions are visible on axial view (ventricles) and on coronal view (sagittal mid-plane). Bottom: results after performing a $3 D$ multimodal non-rigid registration. Anatomical structures are well registered, see ventricles on axial view for instance.

As we have the segmentation of the phantom (grey matter and white matter classes), we can evaluate objectively the registration process. We deform the segmentation volumes as it is described at the beginning of section (3.1). We can assess the quality of the registration by computing overlapping measures (specificity, sensitivity and total performance, see [20] for tutorial) between the initial classes and the deformed classes, once registered with the estimated deformation field. These numerical results are shown on table (1). At the end of grid level 5, we manage to recover up to $95 \%$ of the segmentation, which is a satisfactory result because we use only binary classes (and not fuzzy classes) and a simple linear interpolation scheme. Accordingly to the overlapping measures, we verify that the similarity is increasing as the registration process goes further.

\subsection{Real Data}

We have performed the algorithm on real data (see figure (2). The patient has a cyst and a bone tumor, therefore the multiple interfaces (air/cyst/bone) introduce large distortions that are visible after rigid registration. 


\begin{tabular}{|c|c|cc|}
\hline Registration & Overlap measure & Grey matter & White matter \\
\hline Rigid & sensibility & $74.7 \%$ & $76.6 \%$ \\
& specificity & $93.0 \%$ & $92.8 \%$ \\
& total performance & $87.0 \%$ & $87.6 \%$ \\
\hline Non-rigid & sensibility & $84.7 \%$ & $86.0 \%$ \\
grid level 7 & specificity & $97.2 \%$ & $96.2 \%$ \\
& total performance & $93.2 \%$ & $92.9 \%$ \\
\hline Non-rigid & sensibility & $86.6 \%$ & $86.8 \%$ \\
grid level 6 & specificity & $98.5 \%$ & $97.3 \%$ \\
& total performance & $94.6 \%$ & $93.9 \%$ \\
\hline Non-rigid & sensibility & $87.5 \%$ & $87.0 \%$ \\
grid level 5 & specificity & $98.9 \%$ & $98.0 \%$ \\
& total performance & $95.8 \%$ & $95.3 \%$ \\
\hline
\end{tabular}

Table 1. Numerical evaluation of the multimodal registration method on simulated data. The overlapping measures (specificity, sensitivity and total performance) are computed after rigid registration and at each grid level of the non-rigid registration process.

There are many artafactsin the fMRI acquisition: there has been signal saturation and signal drops (visible in the cyst and in the border of the skull). That illustrates the difficulty of registering clinical data. Although the results are quite difficult to analyze, we can see that the cyst (on the axial view) and the ventricles (on the sagittal view) are better aligned after non-rigid registration.

\section{Conclusion}

In this paper we presented a new method for $3 D$ multimodal non-rigid registration. After rigid registration, we estimate a deformation field with a hierarchical multigrid algorithm. The estimation is performed by minimizing a cost function that is composed of a similarity measure (mutual information) and a regularization term. We have presented results on real data an a numerical evaluation of the algorithm on simulated data.

In the future, we intend to investigate the influence of the similarity measure on the non-rigid registration process. Normalized mutual information [18] and correlation ratio [17] are appealing measure that may give slightly different results. Another perspective is to perform the objective evaluation of this algorithm on a large set of clinical data and study the influence of non-rigid registration on the localization of activated areas.

Acknowledgments. This work has been partly supported by the Brittany Country Council under a contribution to the student grant. The authors would like to thank the SIM laboratory and the Pontchaillou hospital for providing the data. 

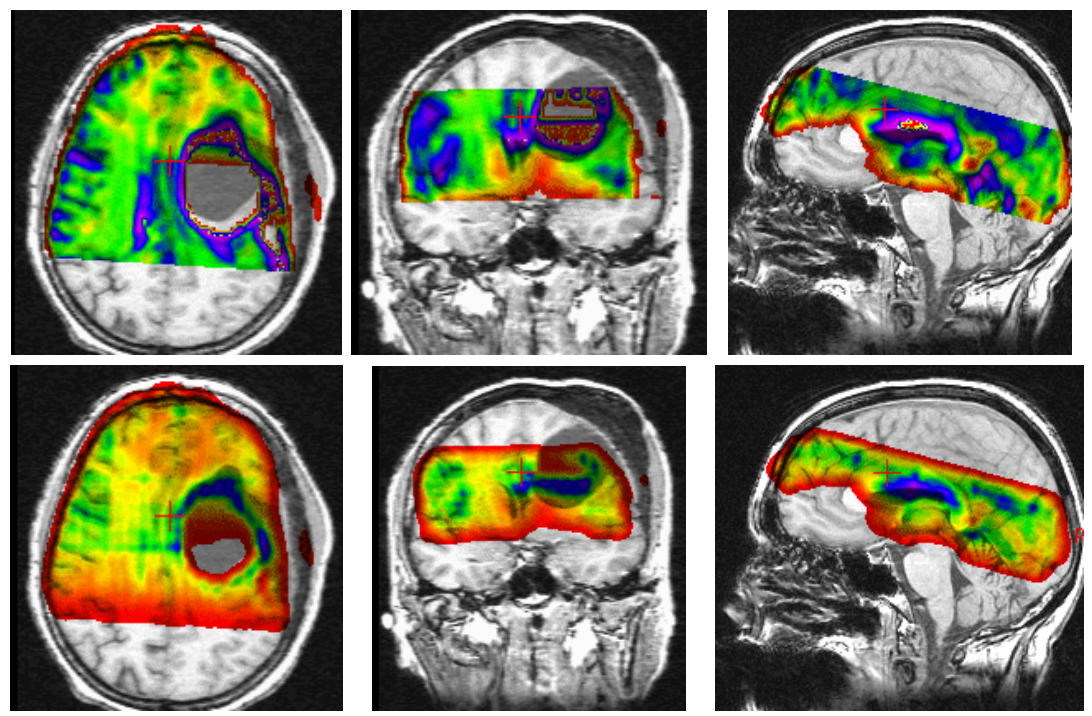

Fig. 2. Results on real data. Top: results of the rigid registration. The multiple artefacts are visible: distortions, signal saturation, signal drops. Bottom: Results of the non-rigid registration. The registration is more accurate, in particular for the ventricles and for the cyst.

\section{References}

1. M. Bastin. Correction of eddy current-induced artefacts in diffusion tensor imaging using iterative cross-correlation. Magnetic Resonance Imaging, 17(7):1011-1024, 1999.

2. F. Bookstein. Principal warps: Thin plate splines and the decomposition of deformations. IEEE PAMI, 11(6):567-585, 1989.

3. A. Collignon, D. Vanderneulen, P. Suetens, and G. Marchal. 3D multi-modality medical image registration using feature space clustering. In Proc. of CVRMed, pages 195-204, 1995.

4. D.L. Collins, A.P. Zijdenbos, V. Kollokian, J.G. Sled, N.J. Kabani, C.J. Holmes, and A.C. Evans. Design and construction of a realistic digital brain phantom. IEEE TMI, 17(3):463468, 1998.

5. W. Enkelmann. Investigations of multigrid algorithms for the estimation of optical flow fields in image sequences. CVGIP, 43(2):150-177, 1988.

6. T. Gaens, F. Maes, D. Vandermeulen, and S. Suetens. Non-rigid multimodal image registration using mutual information. In Proc. of MICCAI, pages 1099-1106, October 1998.

7. A. Guimond, A. Roche, N. Ayache, and J. Meunier. Multimodal brain warping using the demons algorithm and adaptive intensity correction. Technical Report 3796, INRIA, http://www.inria.fr/RRRT/RR-3796.html, November 1999.

8. J. Haselgrove and J. Moore. Correction for distortion of echo-planar images used to calculate the apparent diffusion coefficient. Magnetic Resonance in Medecine, pages 960-964, 1996.

9. P. Hellier, C. Barillot, E. Mémin, and P. Pérez. Medical image registration with robust multigrid techniques. Proc. of MICCAI, pages 680-687, September 1999.

10. M. Horsfield. Mapping eddy current induced fields for the correction of diffusion weighted echo planar images. Magnetic Resonance Imaging, 17(9):1335-1345, 1999. 
11. P. Jezzard and S. Clare. Sources of distortions in functional MRI data. Human Brain Mapping, 8:80-85, 1999.

12. J. Maintz, E. Meijering, and M. Viergever. General multimodal elastic registration based on mutual information. Proc. of Medical Imaging : Image Processing, number 3338 in SPIE Proceedings, pages 144-154, April 1998.

13. J. Maintz and MA. Viergever. A survey of medical image registration. Medical Image Analysis, 2(1):1-36, 1998.

14. CR. Maurer and JM. Fitzpatrick. A review of medical image registration. In Interactive image guided neurosurgery, pages 17-44. American Association of Neurological Surgeons, 1993.

15. E. Mémin and P. Pérez. Dense estimation and object-based segmentation of the optical flow with robust techniques. IEEE Trans. Image Processing, 7(5):703-719, 1998.

16. M. Powell. An efficient method for finding the minimum of a function of several variables without calculating derivatives. The Computer Journal, pages 155-162, 1964.

17. A. Roche, G. Malandain, X. Pennec, and N. Ayache. The correlation ratio as a new similarity measure for multimodal image registration. In Proc. of MICCAI, pages 115-1124, October 1998.

18. C Studholme, D. Hill, and D. Hawkes. An overlap invariant measure of 3D medical image alignment. Pattern Recognition, 32:71-86, 1999.

19. D. Terzopoulos. Image analysis using multigrid relaxation methods. IEEE PAMI, 8(2):129139, 1986.

20. JH. Van Bemmel and MA. Musen. Handbook of medical informatics. Springer, URL : http://www.mieur.nl/mihandbook, 1997.

21. P. Viola and W. Wells. Alignment by maximisation of mutual information. In Proc. ICCV, pages $15-23,1995$.

22. J. West, J. Fitzpatrick, et al. . Comparaison and evaluation of retrospective intermodality brain image registration techniques. Journal of Computer Assisted Tomography, 21(4):554-566, 1997.

23. R. Woods, JC. Mazziotta, and SR. Cherry. MRI-PET registration with automated algorithm. Journal of Computer Assisted Tomography, 17(4):536-546, 1993. 